\title{
Third Delay in Traumatic Brain Injury: Time to Management as a Predictor of Mortality
}

\section{Permanent link}

http://nrs.harvard.edu/urn-3:HUL.InstRepos:39712819

\section{Terms of Use}

This article was downloaded from Harvard University's DASH repository, and is made available under the terms and conditions applicable to Other Posted Material, as set forth at http:// nrs.harvard.edu/urn-3:HUL.InstRepos:dash.current.terms-of-use\#LAA

\section{Share Your Story}

The Harvard community has made this article openly available.

Please share how this access benefits you. Submit a story.

Accessibility 


\section{TABLE OF CONTENTS}

ABSTRACT 


\begin{abstract}
Background: Traumatic brain injury (TBI) is a global epidemic with an increasing incidence in low-and-middle income countries (LMICs). The time from arrival to the hospital to receiving appropriate treatment ("third delay") can vary widely in LMICs, though its association with mortality in TBI remains unknown.

Methods: A retrospective cohort analysis with multivariable logistic regression was conducted with the Towards Improved Trauma Care Outcomes in India database, which contains data from 4 urban trauma centers in India from 2013-2015.

Results: There were 6,278 TBIs included in the cohort. The median age was 39 (IQR 27-52) and 81\% of patients were male. The most frequent mechanisms of injury were road traffic accidents (52\%) and falls (34\%). A majority of cases were transfers from outside facilities (79\%). In-hospital 30-day mortality was $27 \%$; of patients who died, $21 \%$ died within 24 hours of arrival. The median third delay was 10 minutes (IQR $0-60$ minutes); $34 \%$ of cases had moderate third delay (10-60 minutes) and 22\% had extended third delay (61+ minutes). Overall 30-day mortality was associated with moderate third delay $(\mathrm{OR} 1.3, \mathrm{p}=0.001)$ and extended third delay $(\mathrm{OR} 1.3, \mathrm{p}=0.001)$ after adjustment by pertinent co-variates. This effect was pronounced for 24-hour mortality: moderate and extended third delay were independently associated with odds ratios of 3.4 and 3.8 for 24-hour mortality, respectively (both $\mathrm{p}<$ $0.001)$.
\end{abstract}

Discussion: Third delay is associated with early mortality in TBI and represents a target for process improvement in urban trauma centers. 


\section{Acknowledgements}

This work was made possible by the material and education support of many. I thank Harvard Medical School, and in particular Ms. Kari Hannibal, for providing travel funding. I thank the HMS IRB office for their support in study design. I thank my mentors at HMS, especially Dr. Kee Park (Harvard Medical School) and Dr. Blake Alkire (MEEI), for support on study design and for mentoring me before and during when I was in India. Dr. Park's kind mentorship through my four years of medical school raised my interest in global surgery and inspired me to attempt this project and find funding for travel. I also thank Dr. Nakul P. Raykar (BIDMC), whose prior work with the TITCO database and the trauma group in Mumbai provided me with motivation for this study.

I thank the King Edward Memorial Hospital (KEMH) and Lokmanya Tilak Municipal General Hospital (LTMGH) leadership and staff for allowing me to work at their sites. In particular, I would like to thank Drs. Vineet Kumar (LTMGH), Monty Khajanchi (KEMH), and Nobhojit Roy (New Delhi government) for their tireless material support on my project. Drs. Kumar and Khajanchi provided thoughtful and tireless critiques of this work to continually improve it and make it more applicable to real-world TBI patients. I acknowledge Dr. Martin Gerdin, $\mathrm{PhD}$ (Karolinska Institute) for his contributions towards designing statistical analysis for this study. Finally, I thank the curators of the TITCO database for maintaining the database with rigor and integrity, allowing for this and other analyses. 


\section{Disclosures}

None of the authors have disclosures to support. 


\section{Introduction}

Traumatic brain injury (TBI) encompasses a diverse set of pathologies including intracranial bleeding and brain swelling amongst others that is estimated to affect up to 69 million people annually. ${ }^{6}$ Its incidence continues to rise in low- and middle-income countries (LMICs) and presents a significant public health epidemic, as it is often characterized by high morbidity and mortality rates, as well as severe long-term neurological sequelae. In particular, mortality from TBI has not appreciably decreased in over two decades in India. ${ }^{4,14,26}$ Further, it can limit patients' abilities to participate socially and economically within society. ${ }^{4}$

The causes of TBI have undergone rigorous study. Road traffic accidents (RTAs) are the leading cause of TBI in LMICs in part due to rapid urbanization without concomitant improvements in road safety regulations and public knowledge of habits that predispose towards TBI. ${ }^{14,26}$ In India, a multitude of factors have been associated with the high observed rates of TBI, including low helmet and seat-belt usage; high rates of drink-driving; and unsafe roads. $^{2,12}$

Mortality and morbidity largely depend on the time required for patients to access care. ${ }^{21,33}$ Minimizing this figurative "golden hour" has been a priority for various health systems ${ }^{24}$ Various factors can delay the evaluation and management of TBI. These include uncertainty in determining where to seek care after an injury, inefficiency in transportation to the appropriate hospital and transfers between hospitals; workforce and equipment shortages; and inefficient hospital processes and protocols. ${ }^{8,21,25,33}$ Patients' neurological conditions can deteriorate during these delays, contributing to the high rates of irreversible damage or death seen in this population. Borrowing from the maternal health literature, First and second delays represent time from injury to seeking care and time from the decision to seek care to arrival at a hospital with capacity to manage trauma, respectively. ${ }^{3}$ Third delay is the time from arrival to the hospital and management by trained medical professionals. ${ }^{16}$

While reducing all delays is vital to reduce the morbidity associated with TBI, third delay represents a metric that trauma systems may unilaterally improve through resource allocation and protocol implementation for suspected TBIs. Using time between arrival to a hospital and medical assessment as a proxy for the third delay, we describe and characterize the third delay and its association with mortality at four major Indian trauma centers. The objective of this study was to identify the impact of third delay on the primary outcomes: overall 30-day mortality, early mortality (within 24 hours), and late mortality (after 24 hours and within 30 days). 


\section{Methods}

\section{Study Design and Context}

This was a retrospective cohort study conducted in India with the multi-center Towards Improved Trauma Care Outcomes (TITCO) database. This database contains data prospectively collected by trained coders and validated from all admitted trauma patients at four sites, one each within Delhi and Kolkata as well as two within Mumbai, from 2013-2015. ${ }^{9,20}$ All included sites are government-funded centers that provide free to highly subsidized care and contain dedicated neurosurgical departments. All sites were adjacent to major roadways within their respective cities.

The unique features of the TITCO database make it a valid and significant one to study. This database contains information for a population that is nationally representative of urban Indian residents, who are underrepresented in global literature on trauma. This database allows for the study of TBI in LMIC's with high statistical power and validity, and is created and curated by trauma surgeons, who have included variables relevant to the study of TBI, including transfer status, temporal delays, presenting vital signs, diagnoses, procedures, and outcomes amongst others.

The workflow of these centers begins with the arrival of an ambulance, police car, or lay vehicle carrying a patient to the trauma bay. No formal prehospital notification system currently exists at these centers. The vehicle's operator registers the patient with the casualty officer, who triages patients based on clinical history. Casualty officers are usually anesthesiology or surgery resident trainees. When a transportation trolley is made available, the patient is brought into the trauma ward by hospital support staff, and the on-call surgery and anesthesiology resident trainees perform the initial evaluation. The four sites included in TITCO range from having a dedicated 14-bed trauma ward to no dedicated trauma unit. When patients are transferred from outside facilities, they may be accompanied by the results of laboratory and imaging studies done at the outside center.

\section{Case Coding}

Each study site had a dedicated research assistant serving as a coder. Coders worked in 8 hour shifts that cycled through morning, evening, and night rotations to ensure that patients arriving at all times were directly observed and recorded. The data of patients who presented to the hospital outside of a coder's shift was extracted from medical records during a coder's shift. Quarterly quality checks were conducted. During these checks, a random sample of 1- 
$5 \%$ of recorded entries were compared with medical records, and coder recordings demonstrated concordance with patient records.

\section{Inclusion and Exclusion Criteria}

The inclusion criteria for this study included adults (18 years or older) and the presence of a CT-diagnosed TBI (including International Classification of Disease 10 codes s06.1-s06.9) with or without other concomitant bodily injuries. The exclusion criteria included unrecorded time of arrival, unrecorded time of first vitals measurement, and unknown transfer status (Figure 1). These criteria yielded 6,278 TBI cases; of these, 1,521 (24\%) cases were directly observed by coders and the remainder were extracted from patient charts.

\section{Co-variates}

Variables obtained from the TITCO database included the demographic variables of age and gender; clinical characteristics such as Glasgow Coma Scale (GCS) score, Injury Severity Score (ISS), and CT-diagnosed injuries; logistical values such as time and mechanism of injury, mode of transport to the hospital, whether the patient was an out-of-hospital transfer, time of arrival to the hospital, time of first vital signs measurement, and time of CT; whether the patient was directly observed by a coder; and the outcomes of in-hospital death and time to death. Co-variates were binned into physiologically relevant variables as previously described. Glasgow coma scale score was binned as previously described into mild (13-15), moderate (9-12), and severe (3-8); ISS was binned as previously described into mild $(<9)$ moderate $(9-15)$, severe (16-25), and profound $(>25)$. Time of arrival was binned into two hour segments. Third delay was categorized as minimal delay (from the absolute minimum to the median value), moderate delay (from the median to the $3^{\text {rd }}$ quartile value), and extended delay (values above the $3^{\text {rd }}$ quartile). Other delays were categorized as moderate and extended using the same methodology for secondary analyses. All delays were rounded to the nearest minute.

Data Analysis

Descriptive statistics were calculated to quantify presenting patient demographics, injury severity, transfer status, mechanism of injury, timing of injury, and delays along the spectrum of care. The time between arrival to the hospital and measurement of vital signs by physicians within the trauma ward was utilized as a proxy to calculate the third delay since medical decision-making is often initiated based on hemodynamic and respiratory parameters informed by basic vital signs. 
The primary analysis of this study was to determine whether third delay was associated with 30-day overall, early, and late mortality. Sensitivity analysis was conducted for the primary analysis to determine whether effects were modified by the coding method of the case (directly observed by coder vs extracted from medical record). This was conducted by the addition of interaction terms between these variables within the multivariable logistic regression model. Secondary analyses were conducted to assess the impact of other delays, namely time from injury to arrival and time from management to computed tomography (CT), on overall 30-day mortality, early mortality, and late mortality.

\section{Statistical Analysis}

All analyses were conducted on The R Project for Statistical Computing version 3.3.1. Descriptive statistics were first calculated. Multivariable logistic and linear regression analyses were conducted to calculated adjusted odds ratios for primary and secondary analyses. Minimal third delay was used as the reference category. Interaction terms were incorporated into models to query the presence of effect modification. All regression analyses are presented as adjusted odds ratios with $95 \%$ confidence intervals. The cut-off of a probability value $(\mathrm{p})<0.05$ was pre-determined to be the threshold for statistical significance.

Missing co-variate data for regressions were defined as separate levels within each categorical variables and included in multivariable logistic regression analyses. Missing outcome data for secondary analyses was removed with pairwise elimination separately for each secondary analysis.

\section{Ethics Permissions}

Ethical approval, including a waiver of informed consent, was obtained by all hospitals that enrolled patients. These hospitals and their corresponding ethics committee reference numbers were the All India Institute of Medical Sciences (EC/NP-279/2013 RP-Ol/2013), Jai Prakash Narayan Apex Trauma Center (IEC/279), Lokmanya Tilak Municipal General Hospital (IEC/11/13), and the Institute of Post-Graduate Medical Education and Research at the Seth Sukhlal Karnani Memorial Hospital (IEC/279). This study was exempt from formal Institutional Review Board review by Harvard Medical School (IRB 17-2035). All investigators who conducted data manipulation and analysis utilizing the de-identified and de-personalized TITCO data required approval by the TITCO consortium. 


\section{Results}

\section{Case Characteristics}

The median age was 39 (IQR 27 - 52) and 81\% of cases were male (Table 1). Road traffic accidents and falls were the most common mechanisms of injury, representing $52 \%$ and $34 \%$ of all recorded injuries, respectively. The most frequent TBIs noted on CT were parenchymal contusion (54\%), subdural hematoma (38\%), subarachnoid hemorrhage (23\%), and epidural hematoma (21\%). Patients were most commonly transported by ambulance (82\%), and a majority of TBIs in the cohort had been transferred from an outside facility (79\%). On arrival, patients presented with median GCS score of 11 (IQR 7 - 15) and median ISS score of 10 (IQR 9 - 16). In-hospital 30-day mortality was $27 \%$, and of these, $21 \%$ of cases died within 24 hours of arrival.

\section{Variations in Delay and Mortality}

There was considerable variation in the delays involved with seeking and attaining care (Table 2). The time from injury to arrival was available for $94 \%$ of cases. The median time from injury to arrival to hospital with trauma facilities 11 hours (IQR: 4 - $42 \mathrm{hr}$ ). The median third delay was 10 minutes (IQR 0 - $60 \mathrm{~min}$ ). The median time from management to CT was 50 minutes (IQR 20 - $110 \mathrm{~min})$. Third delay was categorized as minimal (44\%, 0-10 min), moderate $(34 \%, 11-60 \mathrm{~min})$, and extended $(22 \%, 61+\min )$. Relative to direct admissions, transfers from outside facilities were independently associated with moderate third delay $(\mathrm{OR} 1.7,95 \%, \mathrm{p}=0.004)$ and extended third delay (OR 1.8, $\mathrm{p}<0.001)$ compared to the minimal delay group.

Moderate and extended third delays were associated with mortality for TBI patients on multivariable analysis (Table 3). Overall 30-day mortality was associated with both moderate delay (OR 1.3, $\mathrm{p}=0.001)$ and extended delay (OR $1.3, \mathrm{p}=0.001$ ). These associations were especially pronounced for 24-hour mortality: moderate and extended third delays had adjusted odds ratios of 3.4 and 3.8 for 24-hour mortality, respectively (both $\mathrm{p}<0.001$ ). This effect was not observed for mortality beyond 24 hours. Case coding method (direct observation vs. medical record review) acted as an effect modifier of the relationship between third delay and mortality despite cases encoded utilizing either method did not have different demographic profiles, time of arrival, clinical severity, or mortality (Table 4). Further, directly observed cases had an adjusted mean decrease in third delay of 31 minutes $(\mathrm{p}<0.001)$ and were less likely to have missing GCS (OR 0.46, $\mathrm{p}<0.001)$ and blood pressure (OR 0.21, $\mathrm{p}<0.001)$ compared to patients coded through review of medical records. 
Secondary analyses revealed that extended delays in time from injury to arrival were associated with lower overall 30-day and late mortality, and that extended delay in time to CT was associated with lower early mortality (Table 5).

\section{Discussion}

This is the first study to quantify third delay and its effects on mortality for TBI utilizing a multicentric approach in an LMIC. We find considerable variation in third delay and observe an association between third delay and inhospital mortality. Half of the TBI cases in this cohort had a third delay over 10 minutes. During this delay, patients can neurologically deteriorate while waiting for care, and unlike patients with major bodily injuries, worsening neurological damage may not be as readily observed visually, in contrast to external bleeding, burns, major fractures, etc. The causes of third delay may encompass many events along the path from the arrival of an ambulance to the evaluation by a physician. A stretcher or support staff may not be available to transport the patient into the hospital, registering a patient prior to assessing them may take time, physicians may not be notified about a new patient, and there may not be a consistently followed protocol to assess the patient in place. Reasons for third delay in previous studies have included workforce and equipment shortages, gaps in workforce training, and inconsistently followed protocols. ${ }^{7}, 16$ Third delay is not unique to LMICs as significant variance in delays in management of high acuity patients has been identified in high-income countries (HICs). ${ }^{11,13,19}$

We identify moderate and extended third delays for TBI patients as independent predictors of 30-day mortality, and in particular for 24-hour mortality. Third delays for obstetric care in other LMICs have been implicated in markedly increased risk for adverse outcomes. ${ }^{3,5}$ Third delay reflects the efficiency of trauma protocol and workforce capacity, which physicians and hospital administrators have the ability to modify. Pre-hospital delays are equally important, but often require change in non-health sector policy, such as increased funding for infrastructure. Further, time from management to $\mathrm{CT}$ and if necessary, to surgery, are also important parameters to optimize, but may require investments in equipment in both LMICs and HICs that require funding at a policy level. ${ }^{1,10,23,32}$

The presence of a coder directly observing in the trauma bay was an effect modifier of this association between third delay and mortality. Providers' may have modified their approach to evaluation and management with a coder present, creating a Hawthorne Effect that might encourage providers to adhere more strictly to protocol. In the presence of a coder, missing vital signs data were less frequent, though this may be explained by changes in behavior or conversely, casualty officers' taking vital signs but not recording them in a chart. This effect has been observed in various studies of physician adherence to guidelines and productivity. ${ }^{15,17,18,31}$ The coders likely did not 
actively influence providers since they were not medical professionals themselves and were not involved in the generation of hypotheses related to variables they coded.

The majority of patients were transferred from facilities that they may not have been able to afford, that were unequipped or did not have qualified staff to manage TBIs, or that were over-capacity to admit the patient. ${ }^{27}$ These transferred patients were more likely to experience a third delay than direct admissions, which may reflect differing protocols in evaluating transferred and directly admitted patients; anticipation of ancillary information from the transferring center such as laboratory and imaging results; and implicit provider assumptions that a transferred patient may have already been adequately stabilized. Similar effects of third delay on mortality were observed for both direct admissions and transferred patients, indicating the importance of this metric regardless of admission type.

Secondary analyses of the delays between time from injury to arrival and time from management to CT revealed counterintuitive associations between delay and mortality. Extended delay in arrival was associated with decreased overall mortality in this cohort. Patients and onlookers may readily recognize a critical injury and immediately seek care, while patients with milder injuries may not seek care until symptoms become more noticeable. Further, the most severe injuries may cause death on-scene, which would not be captured in these data. A study conducted in an HIC observed that TBI patients that present late after injury are often less severe cases with lower polytrauma. ${ }^{25}$ Previous studies in LMICs and HICs showed wide variability in time to arrival and inconsistency in whether delays in arrival affect mortality, reflecting the importance of considering the epidemiological profile, access to care, and surgical capacity of each study's location. ${ }^{8,25,33}$ Time to CT also varied widely and delays tended to be longer than those observed in HICs. ${ }^{21}$ Extended delay in time from the initiation of trauma management to CT was associated with decreased early mortality. This may reflect appropriate triage in the trauma bay such that patients who are clinically more severe are rapidly brought to CT; it likely also reflects that critical patients may have died prior to receiving a $\mathrm{CT}$.

This study has pertinent limitations. The generalizability of this study is limited to urban trauma centers near major transportation routes that have epidemiological patterns of TBI observed in LMICs. The sites included within TITCO are all government-funded, which may enrich for patients of lower socioeconomic status and thus may implement different trauma protocols than private hospitals. We also do not include analysis of other co-morbid traumas or chronic conditions, which are significant determinants of outcome for TBI. 
Patients were included if they had a CT-diagnosed intracranial injury. This may have selected for more severe patients, as patients who presented more mildly may not have received a CT and certain patients with mild TBI may not have manifested an injury visible on CT. Data fidelity may differ between cases that were recorded with direct observation and medical record review since when present, coders directly witnessed vital signs measurements, while it is possible the physicians measuring vitals may not have recorded the exact measurements they recorded into the medical record given the primary priority of managing the patient and time limitations. Other delays that were outside of the scope of this study also affect TBI outcomes. For instance, short time from arrival to neurosurgical intervention for operative candidates may impact survival for severe TBI. ${ }^{22}$ Early initiation of inpatient rehabilitation improves recovery from severe TBI in certain populations ${ }^{29}$ Finally, the gold standard outcome to study TBI is long-term functional outcome, and the TITCO database does not contain information on functional outcome by the time of discharge or any follow-up data after the hospitalization.

Causes for third delay may differ by center and country and should be queried to develop approaches towards risk reduction customized to specific challenges at each trauma center. However, efforts to decrease time intervals in emergency settings need not necessitate additional resources, but rather take advantage of efficient resource allocation that is evaluated rigorously. ${ }^{28,30}$ The finding of a Hawthorne effect in this cohort also suggests the mere presence of an observer decreases third delay, so self-monitoring by individual departments could both identify gaps in care while reducing delay.

Access to care depends not only the availability of a trauma center, but timely care once there. Though the impact of third delay on mortality requires further validation, it represents an actionable process metric that trauma systems may reduce to improve TBI outcome in LMICs, where TBI remains a significant contributor to mortality, especially amongst young people. ${ }^{14}$

\section{Conclusion}

Considerable variation exists in the delay between arrival to the hospital and management ("third delay") in the care of TBI patients. Third delays over 10 minutes and 60 minutes are associated with 3.4-fold and 3.8-fold increased odds of early mortality in TBI patients, respectively. Transferred patients may be especially susceptible to third delays. Our findings implicate third delay as a potential target for intervention to reduce mortality associated with TBI, especially in LMICs. 


\section{Response to Reviewer}

I thank the reviewer for their thoughtful comments and time on my proposal. They reviewer raised significant points in my proposal that I addressed to the best of my ability in my thesis. Below is an itemized response list:

1) "Suggest a bit more about the unique nature of the study why this data set is important" Thank you for this comment. I have included a paragraph in my methods section solely on this topic (Page 7, Paragraph 2).

2) A bit more on the limitations. Lack of generalizability and links to other co-morbids access issues and longterm disability.

Thank you for this comment. I have included a significant list of discussions and their implications in the discussion (Page 12, Paragraphs 3-4). Further, I have cited manuscripts that have addressed some of these topics.

3) Of interest would be to use the dataset to define those with the highest likelihood of doing poorly with delay. This might inform care policy and help prioritize resources in that environment and others that are similar.

Thank you for this comment. We have identified that patients who are transferred are at a higher risk of delay, and believe this would help prioritize resources in this environment. We believe there are logical next steps from this finding that may include communication from the transferring hospital and a dedicated protocol for receiving transferred patients. 


\section{References}

1. Alkire BC, Raykar NP, Shrime MG, Weiser TG, Bickler SW, Rose JA, et al: Global access to surgical care: a modelling study. Lancet Glob Health 3:e316-323, 2015

2. Annadurai K, Mani G, Danasekaran R: Recurring tragedy of road traffic accidents in India: Challenges and opportunities. Indian J Crit Care Med 19:434-435, 2015

3. Assarag B, Dujardin B, Delamou A, Meski FZ, De Brouwere V: Determinants of maternal nearmiss in Morocco: too late, too far, too sloppy? PLoS One 10:e0116675, 2015

4. Brooks JC, Strauss DJ, Shavelle RM, Paculdo DR, Hammond FM, Harrison-Felix CL: Long-term disability and survival in traumatic brain injury: results from the National Institute on Disability and Rehabilitation Research Model Systems. Arch Phys Med Rehabil 94:2203-2209, 2013

5. David E, Machungo F, Zanconato G, Cavaliere E, Fiosse S, Sululu C, et al: Maternal near miss and maternal deaths in Mozambique: a cross-sectional, region-wide study of 635 consecutive cases assisted in health facilities of Maputo province. BMC Pregnancy Childbirth 14:401, 2014

6. Dewan MC, Rattani A, Gupta S, Baticulon RE, Hung YC, Punchak M, et al: Estimating the global incidence of traumatic brain injury. J Neurosurg:1-18, 2018

7. Forshaw J, Raybould S, Lewis E, Muyingo M, Weeks A, Reed K, et al: Exploring the third delay: an audit evaluating obstetric triage at Mulago National Referral Hospital. BMC Pregnancy Childbirth 16:300, 2016

8. Gale SC, Peters J, Hansen A, Dombrovskiy VY, Detwiler PW: Impact of transfer distance and time on rural brain injury outcomes. Brain Inj 30:437-440, 2016

9. Gerdin M, Roy N, Khajanchi M, Kumar V, Fellander-Tsai L, Petzold M, et al: Validation of a novel prediction model for early mortality in adult trauma patients in three public university hospitals in urban India. BMC Emerg Med 16:15, 2016

10. Ginde AA, Foianini A, Renner DM, Valley M, Camargo CA, Jr.: Availability and quality of computed tomography and magnetic resonance imaging equipment in U.S. emergency departments. Acad Emerg Med 15:780-783, 2008

11. Goodman DM, Srofenyoh EK, Olufolabi AJ, Kim SM, Owen MD: The third delay: understanding waiting time for obstetric referrals at a large regional hospital in Ghana. BMC Pregnancy Childbirth 17:216, 2017

12. Gopalakrishnan S: A public health perspective of road traffic accidents. J Family Med Prim Care 1:144-150, 2012

13. Horwitz LI, Green J, Bradley EH: US emergency department performance on wait time and length of visit. Ann Emerg Med 55:133-141, 2010

14. Hyder AA, Wunderlich CA, Puvanachandra P, Gururaj G, Kobusingye OC: The impact of traumatic brain injuries: a global perspective. NeuroRehabilitation 22:341-353, 2007

15. Kidwai AS, Abujudeh HH: Radiologist Productivity Increases With Real-Time Monitoring: The Hawthorne Effect. J Am Coll Radiol 12:1151-1154, 2015

16. Knight HE, Self A, Kennedy SH: Why are women dying when they reach hospital on time? A systematic review of the 'third delay'. PLoS One 8:e63846, 2013

17. Kovacs-Litman A, Wong K, Shojania KG, Callery S, Vearncombe M, Leis JA: Do physicians clean their hands? Insights from a covert observational study. J Hosp Med 11:862-864, 2016

18. Leonard KL, Masatu MC: Changing health care provider performance through measurement. Soc Sci Med 181:54-65, 2017

19. Mahmoodian F, Eqtesadi R, Ghareghani A: Waiting times in emergency department after using the emergency severity index triage tool. Arch Trauma Res 3:e19507, 2014

20. Mansourati M, Kumar V, Khajanchi M, Saha ML, Dharap S, Seger R, et al: Mortality following surgery for trauma in an Indian trauma cohort. Br J Surg, 2018

21. Marcoux J, Bracco D, Saluja RS: Temporal delays in trauma craniotomies. J Neurosurg 125:642-647, 2016 
22. Matsushima K, Inaba K, Siboni S, Skiada D, Strumwasser AM, Magee GA, et al: Emergent operation for isolated severe traumatic brain injury: Does time matter? J Trauma Acute Care Surg 79:838-842, 2015

23. McLane HC, Berkowitz AL, Patenaude BN, McKenzie ED, Wolper E, Wahlster S, et al: Availability, accessibility, and affordability of neurodiagnostic tests in 37 countries. Neurology 85:1614-1622, 2015

24. Newgard CD, Meier EN, Bulger EM, Buick J, Sheehan K, Lin S, et al: Revisiting the "Golden Hour": An Evaluation of Out-of-Hospital Time in Shock and Traumatic Brain Injury. Ann Emerg Med 66:30-41, 41 e31-33, 2015

25. Raj R, Siironen J, Kivisaari R, Kuisma M, Brinck T, Lappalainen J, et al: Factors correlating with delayed trauma center admission following traumatic brain injury. Scand J Trauma Resusc Emerg Med 21:67, 2013

26. Roozenbeek B, Maas AI, Menon DK: Changing patterns in the epidemiology of traumatic brain injury. Nat Rev Neurol 9:231-236, 2013

27. Roy N, Murlidhar V, Chowdhury R, Patil SB, Supe PA, Vaishnav PD, et al: Where there are no emergency medical services-prehospital care for the injured in Mumbai, India. Prehosp Disaster Med 25:145-151, 2010

28. Storm-Versloot MN, Vermeulen H, van Lammeren N, Luitse JS, Goslings JC: Influence of the Manchester triage system on waiting time, treatment time, length of stay and patient satisfaction; a before and after study. Emerg Med J 31:13-18, 2014

29. Tepas JJ, 3rd, Leaphart CL, Pieper P, Beaulieu CL, Spierre LR, Tuten JD, et al: The effect of delay in rehabilitation on outcome of severe traumatic brain injury. J Pediatr Surg 44:368-372, 2009

30. Theunissen BH, Lardenoye S, Hannemann PH, Gerritsen K, Brink PR, Poeze M: Fast Track by physician assistants shortens waiting and turnaround times of trauma patients in an emergency department. Eur J Trauma Emerg Surg 40:87-91, 2014

31. Usichenko TI, Julich A, Wendt M: 'Yes, we can' utilize the Hawthorne effect to improve postoperative analgesia. Br J Anaesth 110:659, 2013

32. Uthkarsh PS, Gururaj G, Reddy SS, Rajanna MS: Assessment and Availability of Trauma Care Services in a District Hospital of South India; A Field Observational Study. Bull Emerg Trauma 4:93-100, 2016

33. Vaca SD, Kuo BJ, Nickenig Vissoci JR, Staton CA, Xu LW, Muhumuza M, et al: Temporal Delays Along the Neurosurgical Care Continuum for Traumatic Brain Injury Patients at a Tertiary Care Hospital in Kampala, Uganda. Neurosurgery, 2018 


\section{Tables}

Table 1. Patient Demographics and Presenting Details

\begin{tabular}{|c|c|c|}
\hline Age & Median & IQR \\
\hline Years & 39 & $27-52$ \\
\hline Sex & $\mathbf{n}$ & $\%$ \\
\hline Male & 5027 & 80.8 \\
\hline Transfer Status & $\mathbf{n}$ & $\%$ \\
\hline Transfer & 4971 & 79.2 \\
\hline Direct Admission & 1307 & 20.8 \\
\hline Mechanism of Injury & $\mathbf{n}$ & $\%$ \\
\hline RTA* & 3257 & 51.9 \\
\hline Fall & 2130 & 33.9 \\
\hline Railway Injury & 370 & 5.9 \\
\hline Assault & 335 & 5.3 \\
\hline Burn & 8 & 0.1 \\
\hline Other & 147 & 2.3 \\
\hline Unknown & 31 & 0.5 \\
\hline Mode of Transport & $\mathbf{n}$ & $\%$ \\
\hline Ambulance & 4932 & 78.6 \\
\hline Police & 482 & 7.7 \\
\hline Rickshaw/Taxi & 424 & 6.8 \\
\hline Private Car & 369 & 5.9 \\
\hline Other & 5 & 0.1 \\
\hline Unknown & 66 & 1.0 \\
\hline Systolic Blood Pressure & n & $\%$ \\
\hline Hypotensive $(<90)$ & 166 & 2.6 \\
\hline Normotensive & 4508 & 71.9 \\
\hline Hypertensive $(>140)$ & 478 & 7.6 \\
\hline Missing & 1126 & 17.9 \\
\hline $\begin{array}{l}\text { Glasgow Coma Scale } \\
\text { Score }\end{array}$ & $\mathbf{n}$ & $\%$ \\
\hline Mild (13-15) & 2466 & 39.3 \\
\hline Moderate (9-12) & 1238 & 19.7 \\
\hline Severe $(3-8)$ & 2260 & 36.0 \\
\hline Missing & 314 & 5.0 \\
\hline Injury Severity Score & $\mathbf{n}$ & $\%$ \\
\hline Mild $(<9)$ & 223 & 3.6 \\
\hline Moderate (9-15) & 4196 & 66.8 \\
\hline Severe (16-25) & 922 & 14.7 \\
\hline Profound $(>25)$ & 592 & 9.4 \\
\hline Missing & 345 & 5.5 \\
\hline
\end{tabular}

*road traffic accident 
Table 2. Assessment of delays.

\begin{tabular}{|l|c|c|}
\hline Delay & Median & IQR $^{*}$ \\
\hline Time from Injury to Arrival & $11 \mathrm{hr}$ & $4-40 \mathrm{hr}$ \\
Time from Arrival to Management & $10 \mathrm{~min}$ & $0-60 \mathrm{~min}$ \\
Time from Management to CT** & $50 \mathrm{~min}$ & $20-110 \mathrm{~min}$ \\
\hline
\end{tabular}

*interquartile range

** computed tomography 
Table 3. Third delay is associated with overall and 24-hour mortality within hospitalization after adjustment for age, sex, transfer status, coder direct observation vs. chart review, blood pressure, ventilator requirement, GCS, and ISS.

\begin{tabular}{|l|ccc|ccc|}
\hline \multirow{2}{*}{ Outcome } & \multicolumn{3}{|c|}{ Moderate Delay } & \multicolumn{3}{c|}{ Extended Delay } \\
\cline { 2 - 7 } & OR* $^{*}$ & $\mathbf{9 5 \%}$ CI** & p & OR & 95\% CI & p \\
\hline Overall Mortality & 1.29 & $1.10-1.52$ & 0.001 & 1.35 & $1.12-1.62$ & 0.001 \\
Mortality Within 24 Hours & 3.36 & $2.51-4.49$ & $<0.001$ & 3.81 & $2.77-5.25$ & $<0.001$ \\
Mortality After 24 Hours & 1.05 & $0.89-1.25$ & 0.558 & 1.07 & $0.88-1.30$ & 0.517 \\
\hline
\end{tabular}

*odds ratio

** confidence interval 
Table 4. Analysis of the impact of third delay and mortality with addition of interaction terms between direct observation by a coder and moderate as well as extended third delay.

\begin{tabular}{|c|c|c|c|c|c|c|c|}
\hline \multirow{2}{*}{ Case Coding Method } & \multirow{2}{*}{ Outcome } & \multicolumn{3}{|c|}{ Moderate Delay } & \multicolumn{3}{|c|}{ Extended Delay } \\
\hline & & OR* & $95 \% \mathrm{CI} * *$ & $\mathbf{p}$ & OR & $95 \% \mathrm{CI}$ & $\mathbf{p}$ \\
\hline \multirow{3}{*}{$\begin{array}{l}\text { Medical Record } \\
\text { Review }\end{array}$} & Overall Mortality & 1.38 & $1.15-1.66$ & 0.001 & 1.38 & $1.15-1.67$ & 0.001 \\
\hline & Mortality Within 24 Hours & 3.39 & $2.44-4.71$ & $<0.001$ & 3.98 & $2.85-5.54$ & $<0.001$ \\
\hline & Mortality After 24 Hours & 1.14 & $0.93-1.39$ & 0.200 & 1.09 & $0.88-1.33$ & 0.430 \\
\hline \multirow{3}{*}{ Direct Observation } & Overall Mortality & 0.79 & $0.57-1.10$ & 0.169 & 0.78 & $0.35-1.77$ & 0.556 \\
\hline & Mortality Within 24 Hours & 0.95 & $0.51-1.76$ & 0.861 & 0.26 & $0.03-2.16$ & 0.213 \\
\hline & Mortality After 24 Hours & 0.77 & $0.54-1.09$ & 0.140 & 0.97 & $0.42-2.22$ & 0.946 \\
\hline
\end{tabular}

*odds ratio

** confidence interval 
Table 5. Secondary analyses are shown. Extended delay ( $>42$ hours) in time from injury to arrival is associated with less overall and late mortality than no delay; moderate ( $>50$ minutes) and extended $(>110$ minutes) delay in management to CT are associated with lower 24-hour mortality. Age, sex, transfer status, case coding method, blood pressure, ventilator requirement, GCS, and ISS are used as adjustment co-variates. Odds ratios for time from management to $\mathrm{CT}$ are also adjusted by degree of third delay and coder direct observation vs. chart review.

\begin{tabular}{|l|l|ccc|ccc|}
\hline \multirow{2}{*}{ Delay } & \multirow{2}{*}{ Outcome } & \multicolumn{3}{|c|}{ Moderate Delay } & \multicolumn{3}{c|}{ Extended Delay } \\
\cline { 3 - 8 } & & OR* $^{*}$ & $\mathbf{9 5 \%}$ CI*** & p & OR & $\mathbf{9 5 \%}$ CI & p \\
\hline \multirow{2}{*}{ Injury to } & Overall Mortality & 1.02 & $0.86-1.22$ & 0.784 & 0.70 & $0.57-0.86$ & 0.001 \\
Arrival & Mortality Within 24 Hours & 0.97 & $0.72-1.30$ & 0.826 & 0.84 & $0.58-1.22$ & 0.358 \\
& Mortality After 24 Hours & 1.01 & $0.85-1.20$ & 0.891 & 0.75 & $0.62-0.90$ & 0.003 \\
\hline \hline \multirow{3}{*}{ Management to } & Overall Mortality & 0.90 & $0.77-1.07$ & 0.229 & 0.92 & $0.71-1.19$ & 0.509 \\
$\mathrm{CT}^{+}$ & Mortality Within 24 Hours & 0.74 & $0.55-1.00$ & 0.051 & 0.26 & $0.13-0.54$ & $<0.001$ \\
& Mortality After 24 Hours & 0.96 & $0.81-1.14$ & 0.618 & 1.07 & $0.82-1.38$ & 0.636 \\
\hline
\end{tabular}

*odds ratio

** confidence interval

${ }^{+}$computed tomography 


\section{Figures}

Figure 1. Patient inclusion/exclusion flowchart. 12,666 adults from the TITCO database were available for analysis, including 6,451 TBIs. Of these cases, 6,278 met the exclusion criteria (97.3\%).

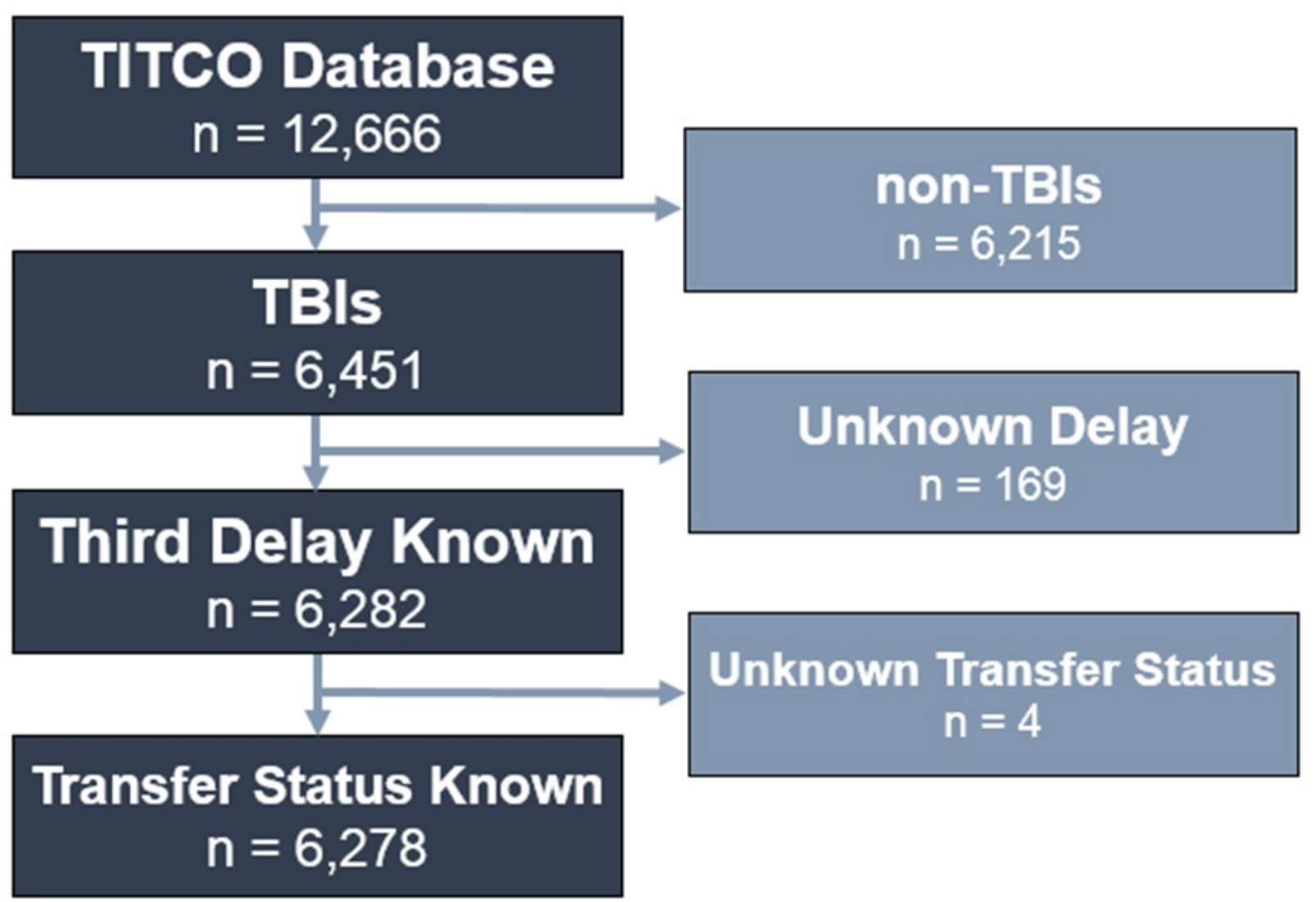

